ANNALES

POLONICI MATHEMATICI

$80(2003)$

\title{
Cyclic coverings of Fano threefolds
}

\author{
by SŁawomir Cynk (Kraków)
}

\begin{abstract}
We describe a series of Calabi-Yau manifolds which are cyclic coverings of a Fano 3-fold branched along a smooth divisor. For all the examples we compute the Euler characteristic and the Hodge numbers. All examples have small Picard number $\varrho=h^{1,1}$.
\end{abstract}

1. Introduction. In $[4,2]$ we constructed a class of Calabi-Yau manifolds that can be realized as a double covering of $\mathbb{P}^{3}$ branched along an octic surface. If the octic was singular we constructed its embedded resolution getting a smooth 3 -fold which contains a smooth (reduced) divisor, equivalent to the -2 multiple of the canonical divisor.

We can generalize this construction studying cycle coverings of Fano 3 -folds. A smooth manifold $Y$ is called Fano if the divisor $-K_{Y}$ is ample; the index of $Y$ is the greatest integer $k$ such that $\frac{1}{k} K_{Y}$ is a Cartier divisor.

The main goal of the paper is to prove the following

Theorem 1. Let $Y$ be a Fano 3-fold of index $k$ and let $d$ be an integer such that $d-1$ divides $k$. Assume that $D$ is a smooth (reduced) divisor in the linear system

$$
\left|-\frac{d}{d-1} K_{Y}\right|
$$

Then there exists a cyclic covering $\pi: X \rightarrow Y$ of order d branched along $D$ such that:

(1) $X$ is a smooth Calabi-Yau manifold,

(2) $e(X)=d e(Y)+\frac{d^{2}}{(d-1)^{2}} K_{Y}^{3}-24 d$,

(3) $h^{1,1}(X)=h^{1,1}(Y)$.

2000 Mathematics Subject Classification: Primary 14B05, 14J30; Secondary 32B10.

Key words and phrases: Calabi-Yau manifolds, cyclic coverings, singularities.

Partially supported by KBN grant no 2P03A 02217.

The author holds a temporary research position at the Institute of Mathematics of the Polish Academy of Sciences. 
Using this theorem we can construct a series of examples of CalabiYau manifolds. The construction is based on the classification of Fano 3folds given by Iskovskikh $([10,11])$ and Mori and Mukai $([12])$. The main difficulty in constructing an explicit example is to find a smooth divisor in the appropriate linear system on the Fano 3-fold from the list. On the other hand the existence of such a divisor follows from [13] so it is easy to make a list of numerical data of 89 Calabi-Yau manifolds. Since the examples with Picard number 1 are well known, we only include examples with Picard number $\geq 2$, i.e. resulting from the list of Mori and Mukai.

For the construction it is not important that the anticanonical divisor is ample; it is enough to assume that the linear system $\left|-\frac{d}{d-1} K_{Y}\right|$ contains a smooth divisor. Moreover we point out that the divisor $D$ need not a priori be irreducible.

In the case of a double octic we produce new examples by allowing certain types of singularities; we can do the same also in the case of cyclic coverings of Fano 3-folds. Again we can define the notion of an admissible blow-up, i.e. a blow-up with smooth center which does not affect the canonical divisor and the first Betti number of the double covering. There are exactly seven types of admissible blow-ups, four in the case of double covering (blow-up of a fourfold and fivefold point and of a double and triple curve). The only new types are blow-ups of a triple, fourfold and fivefold point in a triple covering. A blow-up of a fourfold or fivefold point in a triple covering leads to a singularity that cannot be resolved by admissible blow-ups. For every type of admissible blow-ups we compute the effect on the Euler characteristic of the smooth model of the double covering.

2. Cyclic coverings. In this section we collect some information about cyclic coverings of smooth projective manifolds; the details and proofs can be found in [7].

Let $Y$ be a non-singular complex algebraic variety and let $D=\sum_{i} D_{i}$ be a reduced divisor on $Y$ which is divisible by $d$ as an element of the Picard group, i.e. there exists a line bundle $\mathcal{L} \in \operatorname{Pic} Y$ such that $\mathcal{O}_{Y}(D) \cong$ $\mathcal{L}^{\otimes d}$. Fixing a section $s \in \Gamma\left(\mathcal{L}^{\otimes d}\right)$ whose zero-divisor is $D$ we can define on $\mathcal{O}_{Y} \oplus \mathcal{L}^{-1} \oplus \ldots \oplus \mathcal{L}^{-(d-1)}$ a structure of $\mathcal{O}_{Y}$-module. Let $X \stackrel{\pi}{\rightarrow} Y$ be the spectrum of $\mathcal{O}_{Y} \oplus \mathcal{L}^{-1} \oplus \ldots \oplus \mathcal{L}^{-(d-1)}$. Then $X \stackrel{\pi}{\rightarrow} Y$ is a cyclic covering of $Y$ of degree $d$ branched along $D$.

Proposition 2.1 ([7, Lemma 3.16]).

(1) $\pi_{*} \mathcal{O}_{X} \cong \mathcal{O}_{Y} \oplus \mathcal{L}^{-1} \oplus \ldots \oplus \mathcal{L}^{-(d-1)}$,

(2) $\pi_{*} \Omega_{X}^{1} \cong \Omega_{Y}^{1} \oplus \Omega_{Y}^{1}(\log D) \otimes \mathcal{L}^{-1} \oplus \ldots \oplus \Omega_{Y}^{1}(\log D) \otimes \mathcal{L}^{-(d-1)}$,

(3) $K_{X} \cong \pi^{*}\left(K_{Y} \otimes \mathcal{L}^{d-1}\right)$. 
3. Proof of Theorem 1. Since the map $\pi$ is finite, for $i>0$,

$$
H^{i} \mathcal{O}_{X} \cong H^{i}\left(\pi_{*} \mathcal{O}_{X}\right) \cong H^{i} \mathcal{O}_{Y} \oplus H^{i} \mathcal{L}^{-1} \oplus \ldots \oplus H^{i} \mathcal{L}^{-(d-1)}=0
$$

by the Kodaira vanishing. In particular, we have $H^{1} \mathcal{O}_{X}=0$. Moreover, by Proposition 2.1(3) and the choice of the branch divisor, $K_{X} \cong \mathcal{O}_{X}$. Consequently, $X$ is a Calabi-Yau manifold.

To prove the formula for the Euler characteristic of $X$, observe that

$$
\# \pi^{-1}(y)= \begin{cases}d & \text { for } x \notin D \\ 1 & \text { for } x \in D\end{cases}
$$

Hence,

$$
e(X)=\operatorname{de}(Y)-(d-1) e(D) .
$$

Since $-K_{Y}$ is ample, $H^{i}\left(\mathcal{O}_{Y}\right)=0$ for $i>0$, and consequently, $\chi\left(\mathcal{O}_{Y}\right)=1$. By the Riemann-Roch theorem,

$$
\chi\left(\mathcal{O}_{Y}\right)=\frac{1}{24} c_{1}(Y) c_{2}(Y)
$$

and so

$$
c_{1}(Y) c_{2}(Y)=24
$$

On the other hand, by adjunction,

$$
\begin{aligned}
& c_{1}(D)=-\frac{1}{d-1} c_{1}(Y), \\
& c_{2}(D)=\frac{d}{d-1} c_{1}(Y) c_{2}(Y)-\frac{d^{2}}{(d-1)^{3}} K_{Y}^{3}
\end{aligned}
$$

and finally

$$
\begin{aligned}
e(X) & =d e(Y)-(d-1)\left(\frac{d}{d-1} c_{1}(Y) c_{2}(Y)-\frac{d^{2}}{(d-1)^{3}} K_{Y}^{3}\right) \\
& =d e(Y)-\frac{d^{2}}{(d-1)^{2}} K_{Y}^{3}-24 d .
\end{aligned}
$$

Assertion (3) follows from Proposition 2.1(2) and the Kawamata-Viehweg vanishing theorem ([7, Thm. 6.2]).

4. Cyclic coverings of Fano manifolds with $h^{1,1} \geq 2$. In [12] the numerical data of all Fano 3-folds with $h^{1,1} \geq 2$ were collected; with three exceptions, all these examples have index 1 . The three exceptions have index 2, each leading to two examples of Calabi-Yau manifolds, a double and triple covering. Using those data we have been able to compute the Euler and Hodge numbers of the resulting Calabi-Yau manifolds and compile Table 1 
(in the table we denote a triple covering by $n^{1}$, where $n$ denotes the double covering of the same Fano 3-fold).

Table 1. Coverings of Fano 3-folds with $h^{1,1,}(Y) \geq 2$

\begin{tabular}{|c|c|c|c|c|c|c|}
\hline No. & $K_{Y}^{3}$ & $e(Y)$ & $d$ & $h^{1,1}(X)$ & $e(X)$ & $h^{1,2}(X)$ \\
\hline 1 & -4 & -38 & 2 & 2 & -140 & 72 \\
\hline 2 & -6 & -34 & 2 & 2 & -140 & 72 \\
\hline 3 & -8 & -16 & 2 & 2 & -112 & 58 \\
\hline 4 & -10 & -14 & 2 & 2 & -116 & 60 \\
\hline 5 & -12 & -6 & 2 & 2 & -108 & 56 \\
\hline 6 & -12 & -12 & 2 & 2 & -120 & 62 \\
\hline 7 & -14 & -4 & 2 & 2 & -112 & 58 \\
\hline 8 & -14 & -12 & 2 & 2 & -128 & 66 \\
\hline 9 & -16 & -4 & 2 & 2 & -120 & 62 \\
\hline 10 & -16 & 0 & 2 & 2 & -112 & 58 \\
\hline 11 & -18 & -4 & 2 & 2 & -128 & 66 \\
\hline 12 & -20 & 0 & 2 & 2 & -128 & 66 \\
\hline 13 & -20 & 2 & 2 & 2 & -124 & 64 \\
\hline 14 & -20 & 4 & 2 & 2 & -120 & 62 \\
\hline 15 & -22 & -2 & 2 & 2 & -140 & 72 \\
\hline 16 & -22 & 2 & 2 & 2 & -132 & 68 \\
\hline 17 & -24 & 4 & 2 & 2 & -136 & 70 \\
\hline 18 & -24 & 2 & 2 & 2 & -140 & 72 \\
\hline 19 & -26 & 2 & 2 & 2 & -148 & 76 \\
\hline 20 & -26 & 6 & 2 & 2 & -140 & 72 \\
\hline 21 & -28 & 4 & 2 & 2 & -152 & 78 \\
\hline 22 & -28 & 6 & 2 & 2 & -148 & 76 \\
\hline 23 & -30 & 4 & 2 & 2 & -160 & 82 \\
\hline 24 & -30 & 6 & 2 & 2 & -156 & 80 \\
\hline 25 & -32 & 4 & 2 & 2 & -168 & 86 \\
\hline 26 & -34 & 6 & 2 & 2 & -172 & 88 \\
\hline 27 & -38 & 6 & 2 & 2 & -188 & 96 \\
\hline 28 & -40 & 4 & 2 & 2 & -200 & 102 \\
\hline 29 & -40 & 6 & 2 & 2 & -196 & 100 \\
\hline 30 & -46 & 6 & 2 & 2 & -220 & 112 \\
\hline 31 & -46 & 6 & 2 & 2 & -220 & 112 \\
\hline 32 & -48 & 6 & 2 & 2 & -228 & 116 \\
\hline $32^{1}$ & -48 & 6 & 3 & 2 & -162 & 83 \\
\hline 33 & -54 & 6 & 2 & 2 & -252 & 128 \\
\hline 34 & -54 & 6 & 2 & 2 & -252 & 128 \\
\hline 35 & -56 & 6 & 2 & 2 & -260 & 132 \\
\hline $35^{1}$ & -56 & 6 & 3 & 2 & -180 & 92 \\
\hline 36 & -62 & 6 & 2 & 2 & -284 & 144 \\
\hline 37 & -12 & -8 & 2 & 3 & -112 & 59 \\
\hline 38 & -14 & 2 & 2 & 3 & -100 & 53 \\
\hline 39 & -18 & 2 & 2 & 3 & -116 & 61 \\
\hline 40 & -18 & 4 & 2 & 3 & -112 & 59 \\
\hline 41 & -20 & 8 & 2 & 3 & -112 & 59 \\
\hline
\end{tabular}


Table 1 (cont.)

\begin{tabular}{|c|c|c|c|c|c|c|}
\hline No. & $K_{Y}^{3}$ & $e(Y)$ & $d$ & $h^{1,1}(X)$ & $e(X)$ & $h^{1,2}(X)$ \\
\hline 42 & -22 & 6 & 2 & 3 & -124 & 65 \\
\hline 43 & -24 & 6 & 2 & 3 & -132 & 69 \\
\hline 44 & -24 & 8 & 2 & 3 & -128 & 67 \\
\hline 45 & -26 & 2 & 2 & 3 & -148 & 77 \\
\hline 46 & -26 & 8 & 2 & 3 & -136 & 71 \\
\hline 47 & -28 & 6 & 2 & 3 & -148 & 77 \\
\hline 48 & -28 & 8 & 2 & 3 & -144 & 75 \\
\hline 49 & -30 & 8 & 2 & 3 & -152 & 79 \\
\hline 50 & -32 & 6 & 2 & 3 & -164 & 85 \\
\hline 51 & -32 & 8 & 2 & 3 & -160 & 83 \\
\hline 52 & -34 & 8 & 2 & 3 & -168 & 87 \\
\hline 53 & -36 & 8 & 2 & 3 & -176 & 91 \\
\hline 54 & -36 & 8 & 2 & 3 & -176 & 91 \\
\hline 55 & -38 & 8 & 2 & 3 & -184 & 95 \\
\hline 56 & -38 & 8 & 2 & 3 & -184 & 95 \\
\hline 57 & -38 & 8 & 2 & 3 & -184 & 95 \\
\hline 58 & -40 & 8 & 2 & 3 & -192 & 99 \\
\hline 59 & -42 & 8 & 2 & 3 & -200 & 103 \\
\hline 60 & -42 & 8 & 2 & 3 & -200 & 103 \\
\hline 61 & -44 & 8 & 2 & 3 & -208 & 107 \\
\hline 62 & -46 & 8 & 2 & 3 & -216 & 111 \\
\hline 63 & -48 & 8 & 2 & 3 & -224 & 115 \\
\hline $63^{1}$ & -48 & 8 & 3 & 3 & -156 & 81 \\
\hline 64 & -50 & 8 & 2 & 3 & -232 & 119 \\
\hline 65 & -50 & 8 & 2 & 3 & -232 & 119 \\
\hline 66 & -52 & 8 & 2 & 3 & -240 & 123 \\
\hline 67 & -24 & 8 & 2 & 4 & -128 & 68 \\
\hline 68 & -28 & 8 & 2 & 4 & -144 & 76 \\
\hline 69 & -30 & 10 & 2 & 4 & -148 & 78 \\
\hline 70 & -32 & 10 & 2 & 4 & -156 & 82 \\
\hline 71 & -32 & 10 & 2 & 4 & -156 & 82 \\
\hline 72 & -34 & 10 & 2 & 4 & -164 & 86 \\
\hline 73 & -36 & 10 & 2 & 4 & -172 & 90 \\
\hline 74 & -38 & 10 & 2 & 4 & -180 & 94 \\
\hline 75 & -40 & 10 & 2 & 4 & -188 & 98 \\
\hline 76 & -42 & 10 & 2 & 4 & -196 & 102 \\
\hline 77 & -44 & 10 & 2 & 4 & -204 & 106 \\
\hline 78 & -46 & 10 & 2 & 4 & -212 & 110 \\
\hline 79 & -28 & 12 & 2 & 5 & -136 & 73 \\
\hline 80 & -36 & 12 & 2 & 5 & -168 & 89 \\
\hline 81 & -36 & 12 & 2 & 5 & -168 & 89 \\
\hline 82 & -36 & 14 & 2 & 6 & -164 & 88 \\
\hline 83 & -24 & 16 & 2 & 7 & -112 & 63 \\
\hline 84 & -18 & 18 & 2 & 8 & -84 & 50 \\
\hline 85 & -12 & 20 & 2 & 9 & -56 & 37 \\
\hline 86 & -6 & 22 & 2 & 10 & -28 & 24 \\
\hline
\end{tabular}


5. Singularities. In $[4,2]$ we constructed a family of Calabi-Yau manifolds by considering double coverings of $\mathbb{P}^{3}$ branched along a singular octic. The class of singularities allowed was described by means of admissible blowups, i.e. blow-ups that do not affect the first Betti number and the canonical class of the double covering. For a $d$-sheeted cyclic covering of $Y$ branched along $D$ we can resolve the singularities by taking a blow-up $\sigma: \widetilde{Y} \rightarrow Y$ with a smooth center $C$ (or a sequence of such blow-ups) and considering the $d$-sheeted covering of $\widetilde{Y}$ branched along the divisor $D^{*}:=\sigma^{*} D-d[m / d] \cdot E$, where $E$ is the exceptional divisor of $\sigma$ and $m$ is the multiplicity of $D$ along $C$.

Since $\sigma^{*} D=\widetilde{D}+m E$ (where $\widetilde{D}$ is the strict transform of $D$ ), we have $D^{*}=\widetilde{D}+q E$ with $q \in\{0, \ldots, d-1\}$. For $q>1$ we consider a cyclic covering branched along a non-reduced divisor, and the construction in this case is more complicated (but we shall not describe it any further as we shall in fact never use it in the present paper-for details see [7]).

Since $K_{\widetilde{Y}}=\sigma^{*} K_{Y}+(r-1) E$, where $r$ is the codimension of $C$ in $Y$, we can formulate the following

Definition 5.1. A blow-up $\sigma$ is called admissible if

$$
(d-1)\left[\frac{m}{d}\right]=r-1 .
$$

From the above definition we easily get the following

LEMMA 5.2. We have the following admissible blow-ups:

(1) double coverings:

(a) blow-up of a fourfold point,

(b) blow-up of a fivefold point,

(c) blow-up of a double curve,

(d) blow-up of a triple curve;

(2) triple coverings:

(a) blow-up of a triple point,

(b) blow-up of a fourfold point,

(c) blow-up of a fivefold point.

REMARK 5.3. The blow-ups of type (1a), ., (1d) were described in $[4,2]$. On the other hand, blow-ups of type (2b) and (2c) lead to a multiple curve in a triple covering which cannot be resolved by admissible blow-ups.

Proposition 5.4. If $\sigma: Y_{0} \rightarrow Y$ is a sequence of admissible blow-ups $\left(\sigma=\sigma_{1} \circ \ldots \circ \sigma_{t}\right)$ and $D_{0}$ is a smooth (reduced) divisor divisible by d such that $\sigma\left(D_{0}\right)=D$, then the $d$-sheeted cyclic covering $X_{0}$ of $Y_{0}$ branched along 
$D_{0}$ is a Calabi-Yau manifold. Moreover

$$
e\left(X_{0}\right)=d e(Y)+\frac{d^{2}}{(d-1)^{2}} K_{Y}^{3}-24 d+\sum_{i=1}^{t} \operatorname{eff}\left(\sigma_{i}\right),
$$

where $\operatorname{eff}\left(\sigma_{i}\right)$ is the effect of $\sigma_{i}$, defined by

$$
\operatorname{eff}\left(\sigma_{i}\right)= \begin{cases}36 & \text { if } \sigma_{i} \text { is of type }(1 \mathrm{a}) \text { or }(1 \mathrm{~b}), \\ 14 \operatorname{deg}(\mathcal{L} \mid C)-6 \operatorname{deg}\left(\bigwedge^{2} \mathcal{N}\right) & \text { if } \sigma_{i} \text { is of type }(1 \mathrm{c}) \text { or }(1 \mathrm{~d}), \\ 24 & \text { if } \sigma_{i} \text { is of type }(2) .\end{cases}
$$

REMARK 5.5. If $\sigma$ is a sequence of blow-ups that are not admissible it does not follow in general that $X$ has no smooth model which is a CalabiYau manifold. If $\sigma_{i}$ is a blow-up of a point and $(d-1)[m / d]<2<r-1$, then there can exist a small resolution (e.g., $A_{1}$ point in the cases $d=2$ or $4, A_{3}$ point in the case $d=2$ ).

On the other hand, if $\sigma_{i}$ is a blow-up with $(d-1)[\mathrm{m} / d]>r-1$ then the exceptional divisor cannot be blown down because it is nef along the fibers. Consequently, also in this situation no smooth model of $X$ is a Calabi-Yau manifold.

EXAMPle 1. If $D \subset \mathbb{P}^{3}$ is a sextic with ordinary triple points, then the triple covering of $\mathbb{P}^{3}$ branched along $D$ has a smooth model $\widetilde{X}$ which is a Calabi-Yau manifold with Euler number

$$
e(\tilde{X})=-204+24 \mu,
$$

where $\mu$ is the number of triple points. By [6] a sextic surface can have up to 10 triple points; all the possible sextics with ordinary triple points where classified in that paper.

EXAmple 2. Let $D=D_{1}+D_{2}$ be a sum of two smooth anticanonical divisors in a Fano 3-fold $Y$ intersecting transversally along a smooth curve $C$. Denote by $\widetilde{Y}$ the blow-up of $Y$ along $C$. Let $\widetilde{D}_{i}$ be a strict transform of $D_{i}$. Then $\widetilde{D}_{i}$ is isomorphic to $D_{i}$. The double covering $\widetilde{X}$ of $\widetilde{Y}$ branched along $\widetilde{D}=\widetilde{D}_{1}+\widetilde{D}_{2}$ is a Calabi-Yau manifold. The Euler characteristic $e(\widetilde{Y})$ equals $2 e(\widetilde{Y})-e(\widetilde{D})$. Now, $e(\widetilde{Y})=e(Y)+e(C)$ and $e(\widetilde{D})=2 e\left(D_{1}\right)$. Computations analogous to the proof of Theorem 1 give $e(C)=K_{Y}^{3}, e\left(D_{1}\right)=24$ and finally

$$
e(\tilde{X})=2 e(Y)+2 K_{Y}^{3}-48 \text {. }
$$

Moreover, by [3],

$$
h^{1,1}(\widetilde{X})=h^{1,1}(Y)+1
$$

\section{References}

[1] C. H. Clemens, Double solids, Adv. Math. 47 (1983), 107-230. 
[2] S. Cynk, Double coverings of octic arrangements with isolated singularities, Adv. Theor. Math. Phys. 3 (1999), 217-225.

[3] - Cohomologies of a double covering of a non-singular algebraic 3-fold, Math. Z., to appear.

[4] S. Cynk and T. Szemberg, Double covers and Calabi-Yau varieties, in: Banach Center Publ. 44, Inst. Math., Polish Acad. Sci., 1998, 93-101.

[5] A. Dimca, Betti numbers of hyperplanes and defects of linear systems, Duke Math. J. 60 (1990), 285-294.

[6] S. Endraß, U. Persson and J. Stevens, Surfaces with triple points, preprint math.AG/ 0010163.

[7] H. Esnault and E. Viehweg, Lectures on Vanishing Theorems, DMV Seminar 20, Birkhäuser, 1992.

[8] P. Griffiths and J. Harris, Principles of Algebraic Geometry, Wiley, New York, 1978.

[9] R. Hartshorne, Algebraic Geometry, Springer, 1977.

[10] V. A. Iskovskikh, Fano 3-folds, I, Math. USSR-Izv. 11 (1977), 485-527.

[11] -, Fano 3-folds, II, ibid. 12 (1978), 469-506.

[12] S. Mori and S. Mukai, Classification of Fano 3-folds with $B_{2} \geq 2$, Manuscripta Math. 36 (1981), 147-162.

[13] V. V. Shokurov, Smoothness of a general anticanonical divisor on a Fano variety, Izv. Akad. Nauk SSSR Ser. Mat. 43 (1979), 430-441 (in Russian).

Institute of Mathematics

Jagiellonian University

Reymonta 4

30-059 Kraków, Poland

E-mail: cynk@im.uj.edu.pl

Reçu par la Rédaction le 25.3.2002

Révisé le 25.4.2002 